\title{
Nonlinear Damping of Oscillations in Tidal-Capture Binaries
}

\author{
Pawan Kumar ${ }^{1,2}$ \\ Institute for Advanced Study, Princeton, NJ 08540 \\ Jeremy Goodman \\ Princeton University Observatory, Peyton Hall, Princeton, NJ 08544 \\ Electronic mail: jeremy@astro.princeton.edu
}

Received —

${ }^{1}$ Permanent address: Massachusetts Institute of Technology, Department of Physics, Cambridge, MA 02139. Electronic mail: pk@brmha.mit.edu

${ }^{2}$ Alfred P. Sloan Fellow \& NSF Young Investigator 


\begin{abstract}
We calculate the damping of quadrupole f- and low order g-modes (primary modes) by nonlinear coupling to other modes of the star. Primary modes destabilize high degree g-modes of half their frequency (daughter modes) by 3 -mode coupling in radiative zones. For sunlike stars, the growth time $\equiv \eta^{-1} \approx 4 E_{0,42}^{-1 / 2}$ days, where $E_{0,42}$ is the initial energy of the primary mode in units of $10^{42} \mathrm{erg}$, and the number of daughter modes $N \sim 10^{10} E_{0,42}^{5 / 4}$. The growth rate is approximately equal to the angular frequency of the primary mode times its dimensionless radial amplitude, $\delta R / R_{*} \approx 0.002 E_{0,42}^{1 / 2}$. Although the daughter modes are limited by their own nonlinearities, collectively they absorb most of the primary mode's energy after a time $\sim 10 \eta^{-1}$ provided $E_{0}>10^{40} \mathrm{erg}$. This is orders of magnitude smaller than usual radiative damping time. In fact nonlinear mode interaction may be the dominant damping process if $E_{0} \gtrsim 10^{37} \mathrm{erg}$. These results have obvious application to tidally captured main sequence globular cluster stars of mass $\geq 0.5 M_{\odot}$; the tidal energy is dissipated in the radiative core of the star in about a month, which is less than the initial orbital period.

Nonlinear mode coupling is a less efficient damping process for fully convective stars, which lack g-modes. In convective stars most of the tidal energy is in the quadrupole f-modes which nonresonantly excite high order p-modes of degree 0,2 , and 4 . The resultant short wavelength waves are more efficiently dissipated. The nonlinear damping time for f-modes is shown to be proportional to $1 / E_{0}$; this damping time is about 30 days for $E_{0} \approx 10^{45} \mathrm{erg}$ expected in tidal captures. However, at such a large energy the system is very nonlinear: four-mode and higher-order couplings are as important as three-mode couplings.
\end{abstract}




\section{Introduction}

Tidal interactions in highly eccentric close binary systems primarily excite low order quadrupole gravity modes (g-modes) and the fundamental mode (f-mode), because these modes have periods comparable to the periastron passage time and their eigenfunctions have the largest overlap with the tidal forcing field [Kumar, Ao, \& Quataert (1995)]. The dissipation time scale for these modes, for main sequence stars, are $10^{4}$ years or greater. A long damping time typically leads to a larger mode amplitude for modes that are subjected to repeated tidal forcing, but perhaps more interestingly offers the possibility that in tidal interactions energy can be transferred from the modes to the orbit as well as from the orbit to the modes, causing a chaotic evolution of the orbit Mardling (1995) in some cases. In the case of tidal capture binaries, this process can lead to ionization of the system in subsequent encounters [Kochanek (1992), Mardling (1995).]

Given the large amplitudes of modes in close binary systems, one expects nonlinear interactions amongst modes carrying most of the energy and others, which are tidally not excited, to be an important process of draining energy from the primary modes. We investigate this process and assess its significance in close binary systems.

The next section describes the basic equations for mode couplings and discusses the most significant nonlinear process for energy transfer from modes of a radiative star. In section $\S 3$ we discuss the nonlinear mode couplings for a convective star. Applications to tidal capture are discussed in $\S 4$, and our main results are summarized in $\S 5$. The details of the calculation of nonlinear coupling coefficients are presented in Appendix A.

\section{Nonlinear Interactions: Stars with Radiative Zones}

The leading-order nonlinear interactions of stellar modes are 3-mode couplings. These can be derived by expanding the Lagrangian density for adiabatic motions in powers of the fluid displacement. If the expansion is truncated at second order, the standard Euler-Lagrange procedure yields linear equations of motion, which one solves for the normal modes. When the third-order parts of the Lagrangian density are retained, the equations of motion for the mode amplitudes contain quadratic terms:

$$
\ddot{A}_{\alpha}+\omega_{\alpha}^{2} A_{\alpha}=-\omega_{\alpha}^{2} \sum_{\beta, \gamma} \kappa_{\alpha \beta \gamma} A_{\beta} A_{\gamma},
$$

where $\omega_{\alpha} \& A_{\alpha}$ are mode frequency and amplitude. The 3-mode coupling coefficient, $\kappa_{\alpha \beta \gamma}$, is completely symmetric in the three indices. It is obtained by substituting the normal mode expansion of fluid displacement into the cubic Lagrangian density, and integrating over the stellar interior (see Appendix A). Since our eigenfunctions are normalized to unit 
energy, the energy in mode $\alpha$ is $A_{\alpha}^{2}$. Note that equation (1) with its right side set to zero describes free normal modes of oscillation. We could represent tidal forcing by adding a term independent of the amplitudes, but in this paper we are concerned with the evolution of the modes after forcing has ceased.

We assume that initially all the pulsation energy resides in a few low order modes. If the star has a radiative core, then these modes couple to high degree g-modes and transfer energy away by parametric resonance instability. This is discussed in the next sub-section. Even for completely convective stars nonlinear energy transfer, involving non-resonant 3 -mode interaction with high frequency p-modes, can be an important process and is discussed in $\S 3$.

\subsection{Parametric instability}

Parametric instability arises when a mode is nonlinearly coupled to another mode of approximately twice the frequency, or more generally when two low frequency modes of infinitesimal amplitude couple to a higher frequency mode of finite amplitude. To analyze this case we consider a single triplet interaction involving modes $\alpha, \beta_{1}$ and $\beta_{2}$. Expressing mode amplitudes in the following complex notation

$$
A_{\alpha}=A \exp \left(i \omega_{\alpha} t\right)+\text { c.c., } \quad A_{\beta_{1}}=B_{1} \exp \left(i \omega_{\beta_{1}} t\right)+\text { c.c. }, \quad A_{\beta_{2}}=B_{2} \exp \left(i \omega_{\beta_{2}} t\right)+\text { c.c. },
$$

and substituting this into eq. (1) we obtain the following simplified equations

$$
\begin{aligned}
& \dot{B}_{1}+\gamma B_{1} / 2=i 3 \omega_{\beta_{1}} \kappa_{\alpha \beta_{1} \beta_{2}} A B_{2}^{*} \exp (i \Delta \omega t), \\
& \dot{B}_{2}+\gamma B_{2} / 2=i 3 \omega_{\beta_{2}} \kappa_{\alpha \beta_{1} \beta_{2}} A B_{1}^{*} \exp (i \Delta \omega t),
\end{aligned}
$$

where $\Delta \omega \equiv \omega_{\alpha}-\omega_{\beta_{1}}-\omega_{\beta_{2}}, \gamma$ is the linear dissipation rate of these modes, and the factor of 3 appears for combinatorial reasons. Rapidly oscillating terms as well as second time derivative of mode amplitudes were discarded in deriving these equations. At initial times when the amplitudes of modes $\beta_{1}$ and $\beta_{2}$ are infinitesimal, we can take the amplitude of mode $\alpha$ to be fixed $\left(A_{0}\right)$, and solve the above two equations for the growth rate $(\eta)$ of mode amplitudes, which is

$$
\eta=\frac{1}{2}\left[\sqrt{9 A_{0}^{2} \kappa_{\alpha \beta_{1} \beta_{2}}^{2} \omega_{\beta_{1}} \omega_{\beta_{2}}-(\Delta \omega)^{2}}-\gamma\right] .
$$

The coupling coefficient $\kappa_{\alpha \beta_{1} \beta_{2}}$ is largest when the modes $\beta_{1}$ and $\beta_{2}$ have nearly the same radial order $n$ and spherical-harmonic degree $\ell$, whence $\omega_{\beta_{1}} \approx \omega_{\beta_{2}} \approx \omega_{\alpha} / 2$. We see that parametric instability sets in provided that

$$
(\Delta \omega)^{2}<9 \kappa_{\alpha \beta_{1} \beta_{2}}^{2} A_{0}^{2} \omega_{\beta_{1}} \omega_{\beta_{2}}-\gamma^{2},
$$


Ignoring the damping for now, we obtain a very simple criterion for parametric instability:

$$
|\Delta \omega|<3 \omega_{\alpha} A_{0} \kappa_{\alpha \beta_{1} \beta_{2}} / 2
$$

The product $A_{0} \kappa_{\alpha \beta_{1} \beta_{2}}$ is a dimensionless quantity which is a measure of the nonlinearity of the system. The coupling coefficients are calculated using equation (A17) of Appendix A, which is a somewhat long expression. However, simple scaling argument shows that $\kappa_{\alpha \beta \beta} \sim G^{-1 / 2} R_{*}^{1 / 2} M_{*}^{-1}$ for a low order quadrupole mode coupled to two high order g-modes (where $M_{*}$ and $R_{*}$ are stellar mass and radius), so that $\kappa_{\alpha \beta_{1} \beta_{2}} \approx 10^{-24} \mathrm{erg}^{-1 / 2}$ for a solar-mass main sequence star. This is in good agreement with detailed numerical calculations (Table 1). If the energy $E_{0}=A_{0}^{2}$ in the f-mode is $10^{42}$ erg then parametric resonance is possible provided that we can find a triplet with $\Delta \omega$ less than $0.15 \%$ of the frequency of the f-mode. We discuss below the possibility of finding such resonant triplets involving high degree g-modes.

To put this energy in perspective, note that the dimensionless radial amplitude of the f-mode is $\delta R / R_{*} \approx 2 \times 10^{-3} E_{0,42}^{1 / 2}$, where $E_{0,42} \equiv E_{0} /\left(10^{42} \mathrm{erg}\right)$. Tidal capture is expected to result in motions $\delta R / R_{*} \sim 0.1, E_{0} \sim 10^{45} \mathrm{erg}$. For comparison, the most energetic five-minute oscillations of the Sun have about $10^{28} \mathrm{erg}$, and the total energy in all solar modes is $\approx 10^{33} \mathrm{erg}$ [Goldreich, Murray, \& Kumar (1994)]. It also follows from these scalings that $\Delta \omega / \omega \approx 2 \eta / \omega \approx \delta R / R_{*}$.

The dispersion relation for g-modes is [e.g. Christensen-Dalsgaard \& Berthomieu $(1991)$

$$
P_{n \ell}=\frac{P_{0}}{2 \sqrt{\ell(\ell+1)}}(2 n+\ell-\delta)+\frac{P_{0}^{2}}{P_{n \ell}}\left[V_{1}+\frac{V_{2}}{\ell(\ell+1)}\right] \text {, }
$$

where

$$
P_{0}=\frac{2 \pi^{2}}{\int_{0}^{r_{1}} d r N_{B} / r}
$$

$P_{n \ell}\left(=2 \pi / \omega_{n \ell}=1 / \nu_{n \ell}\right)$ is the mode period, $N_{B}$ is the Brunt-Väisälä frequency, $r_{1}$ is the upper turning point of the mode, $\delta \approx 5 / 6$, and $V_{1}$ and $V_{2}$ are constants of order 0.5 and 6 respectively for $1 M_{\odot}$ main sequence star. Note that mode frequency goes to zero as $n / \ell$ goes to infinity, and the frequency approaches $P_{0}^{-1}$ as $n / \ell$ tends to zero. Furthermore, it follows from equation (8) that the frequencies of modes of large degree are function of $n / \ell$, and that the frequency spacing between modes of adjacent $n$ for a fixed $\ell, \delta \nu_{n \ell}$, is approximately equal to $\nu_{n \ell}^{2} P_{0} / \ell$. The total number of distinct frequencies $\nu_{n^{\prime} \ell^{\prime}}$ for which both $n^{\prime}$ and $\ell^{\prime}$ are of order $\ell$ scales as $\ell^{2}$. These frequencies are distributed between 0 and $N_{B}$. Statistically therefore, given a typical primary frequency $\nu_{\alpha}<2 N_{B}$, the lowest $\ell$ such that $\left|\nu_{n \ell}-\left(\nu_{\alpha} / 2\right)\right|<\delta \nu$ should be $\ell \sim \ell_{\min } \sim \sqrt{\nu_{\alpha} / 2 \delta \nu}$. It is worth noting that the frequency of the $\ell=2 \mathrm{f}$-mode (strongly excited during tidal capture) is in fact less than $2 N_{B}$ in Sun-like stars, so resonant g-modes will exist.

The radiative damping time of long-wavelength g-modes is of the order of the photon diffusion time or thermal time $t_{t h}$, which for a solar type star is about $10^{7}$ years, and it 
decreases as $\ell^{-2}$ with increasing mode degree. (The mode damping time for a completely radiative star decreases much more rapidly with $\ell$ since the outer turning point of g-modes moves outward with increasing degree. However, main sequence globular cluster stars have a convective envelope of thickness $\gtrsim 10 \%$ of the stellar radius, and for these stars the radius of the outer turning point of high-degree g-modes is independent of $\ell$ at fixed frequency, and the damping time scales as $\ell^{-2}$ ). Thus the damping rate of a mode of degree $\ell$ (and $n \sim \ell$ ) is

$$
\gamma_{\ell} \sim \frac{\ell^{2}}{t_{t h}}
$$

Parametric instability can exist only if there are modes that have both $\gamma_{\ell}$ and $|\Delta \omega|$ less than $3 \omega_{\alpha} A_{0} \kappa_{\alpha \beta_{1} \beta_{2}} / 2$ [see eq. (5)]. This dual condition places a lower limit to the value of $A_{0} \kappa_{\alpha \beta_{1} \beta_{2}}$ at which parametric instability occurs, which we now calculate. By the arguments of the last paragraph, the minimum degree at which the resonance condition (7) is met is

$$
\ell_{\min } \approx\left(\frac{1}{A_{0} \kappa_{\alpha \beta_{1} \beta_{2}}}\right)^{1 / 2}
$$

The maximum degree is set by the requirement that mode damping be less than $3 \omega_{\alpha} A_{0} \kappa_{\alpha \beta_{1} \beta_{2}} / 2$, which gives

$$
\ell_{\max } \approx\left(\frac{3 t_{t h} \omega_{\alpha} A_{0} \kappa_{\alpha \beta_{1} \beta_{2}}}{2}\right)^{1 / 2}
$$

Obviously $\ell_{\max } \geq \ell_{\min }$ in order that there exist g-modes resonantly coupled to the primary mode. This leads to the following condition on mode amplitude for parametric instability to operate:

$$
A_{0} \kappa_{\alpha \beta_{1} \beta_{2}} \gtrsim \sqrt{\frac{1}{3 \omega_{\alpha} t_{t h}}}
$$

For a solar-type main sequence star, typically $\kappa_{\alpha \beta_{1} \beta_{2}} \sim 10^{-24} \mathrm{erg} \mathrm{s}^{-1 / 2}, t_{t h} \sim 10^{14} \mathrm{~s}$, and $\omega_{\alpha} \approx 2 \times 10^{-3} \mathrm{rad} / \mathrm{s}$. Therefore, the f-mode must have energy $\gtrsim 10^{36} \mathrm{erg}$ in order to spawn a lower frequency mode via parametric instability. Solar $\mathrm{p}$ and $\mathrm{f}$ modes lie at least seven orders of magnitude below this threshold (see above).

So far we have neglected stellar rotation. Rotation lifts the degeneracy of mode frequencies with respect to the spherical-harmonic order $m$ : thus we have $\nu_{n \ell m}$ instead of $\nu_{n \ell}$. This enlarges the number of modes available for resonant non-linear interactions, including parametric instability. For a star rotating rigidly with angular velocity $\Omega_{0}$ the frequency splitting for high degree g-modes in the rotating frame of the star, $\delta \nu_{\text {rot }} \equiv \nu_{n, \ell, \ell}-\nu_{n, \ell,-\ell}$, is approximately $\Omega_{0} / 2 \pi \ell$, whereas if the mean differential rotation in the radiative interior of the star is $\overline{\Delta \Omega}$, then $\delta \nu_{\text {rot }} \sim \ell \overline{\Delta \Omega} / 2 \pi$ (see Appendix B). Thus for a rigidly rotating star rotational splitting becomes important at mode degree $\ell$ such that $\delta \nu_{\text {rot }} \approx \nu / \ell^{2}$, or $\ell \approx 2 \pi \nu / \Omega_{0} \approx 10^{3}$ for a star like the Sun. For $\ell$ greater than this value one can find modes with frequency within $\Omega_{0} / \ell^{2}$ of some desired value, which is much better than what we get 
for a nonrotating star. Note that even a very small differential rotation in radiative interior leads to a large value of $\delta \nu_{\text {rot }}$. However, in the case of the Sun, where the internal rotation is known from measurements of p-mode frequency splitting, the differential rotation in the radiative interior appears to be close to zero. Nevertheless, in rotating stars one can find modes with smaller values of $|\Delta \omega|$ and so parametric instability persists down to smaller mode energies.

\subsection{Energy transfer to small-scale modes}

The astrophysical importance of the parametric instability depends upon the amplitude at which nonlinearities intervene to stop exponential growth. In simple mechanical systems, it is common for parametric instabilities to saturate because the natural frequency of the daughter mode is a function of amplitude. Although the same mechanism applies in principle to g modes, it does not affect the linear growth rate until the energy in a single daughter mode is comparable to that in the primary mode, as discussed in more detail in Appendix C. Other nonlinearities become important at much smaller amplitudes.

As the energy of the primary mode decreases to balance the increasing energy in all of its parametrically unstable daughters, equations (5) and (6) remain true if we replace the initial amplitude $\left(A_{0}\right)$ with its current value $\left(A_{\alpha}\right)$. Hence both the width of the unstable resonance and the peak growth rate decrease with $A_{\alpha}$. Initially unstable daughters become stable. It can be shown that parametric resonance involving a single daughter mode, where $\beta_{1}=\beta_{2}$, leads to periodic transfer of energy back and forth between the daughter and the primary.

We now discuss the generalization of this result to the more realistic case where a large number of modes $(\beta)$ of angular frequency $\omega_{\beta} \approx \omega_{\alpha} / 2$ are coupled to the mode $\alpha$ of frequency $\omega_{\alpha}$. At present, however, we ignore direct interactions among the daughters themselves, as well as parametric decay of the daughters into "granddaughters." For clarity we write down the equations for modes $\alpha$ and $\beta$ below:

$$
\begin{gathered}
\dot{A}_{\alpha}=i \frac{3}{2} \omega_{\alpha} \sum_{\beta} \kappa_{\alpha \beta \beta} B_{\beta}^{2} \exp \left(-i \Delta \omega_{\beta} t\right), \\
\dot{B}_{\beta}+\gamma_{\beta} B_{\beta}=i 3 \omega_{\beta} \kappa_{\alpha \beta \beta} A_{\alpha} B_{\beta}^{*} \exp \left(i \Delta \omega_{\beta} t\right)
\end{gathered}
$$

where

$$
\Delta \omega_{\beta} \equiv \omega_{\alpha}-2 \omega_{\beta} .
$$

It is straightforward to show from these equations that in the absence of damping the 
sum of the energy in all modes is conserved, i.e.[?

$$
\left|A_{\alpha}\right|^{2}+\sum_{\beta}\left|B_{\beta}\right|^{2}=A_{0}^{2} \equiv E_{0}
$$

Furthermore, it can be shown that nonlinear interactions lead eventually to equipartition of energy among all the coupled modes. Thus the steady state energy of modes is

$$
\left\langle E_{\alpha}\right\rangle=\left\langle E_{\beta}\right\rangle \approx E_{0} / N
$$

where $N$ is the number of modes involved in parametric resonance with mode $\alpha$. Initially, when all the energy is in mode $\alpha$, daughter modes grow exponentially, and the characteristic time for the energy in mode $\alpha$ to halve is of order $\eta^{-1}$ [eq. (5)]. We shall call this energy-halving timescale $t_{\mathrm{nl}}$, since it derives from nonlinear mode couplings.

In more detail, $t_{\mathrm{nl}}$ depends logarithmically on the mean initial energy per daughter mode, $E_{\beta, 0}$. The latter energy has at least the thermal value $k T \sim 10^{-9} \mathrm{erg}$, whence $t_{\mathrm{nl}}<\ln \left(E_{0} / N k T\right) / 2 \eta \approx 50 / \eta$. Since the g-modes may be stochastically excited by macroscopic processes such as semiconvection in the core or convection in the envelope of the star, it is probably more realistic to assume $t_{\mathrm{nl}} \sim 10 / \eta$, or approximately $50 E_{\alpha, 42}^{-1 / 2}$ days.

In the simplified model of eqs. (14)-(15), complete equipartition [eq. (18)] is obtained only on a timescale $\gg t_{\mathrm{nl}}$, because the nonlinear interaction rate decreases in proportion to the primary amplitude; furthermore, phase coherence between the primary and the daughters tends to be lost. Because of damping and because of coupling to granddaughter modes, equipartition may never be achieved in a real star.

We have verified that our model behaves as described by numerically integrating the differential equations (14)-(15), and the results are shown in fig. 1.

We can compare the nonlinear timescale to the timescale for decay of the primary mode by linear radiative damping. The former is

$$
t_{\mathrm{nl}} \approx \frac{10}{\eta} \approx \frac{40}{3 \omega_{\alpha} A_{0} \kappa_{\alpha \beta_{1} \beta_{2}}} \approx \frac{2}{\nu_{\alpha} A_{0} \kappa_{\alpha \beta_{1} \beta_{2}}} .
$$

If the radiative damping time for the primary mode is $1 / \gamma_{\alpha}$, then parametric instability is the dominant damping process for the mode provided that

$$
A_{0} \kappa_{\alpha \beta_{1} \beta_{2}} \gtrsim \frac{2 \gamma_{\alpha}}{\nu_{\alpha}}
$$

${ }^{3}$ Strictly speaking, the conserved energy includes terms of cubic and higher order in the amplitudes. In a hamiltonian formalism, the latter terms are responsible for mode coupling and for transfer of energy among resonant linear modes. For a weakly nonlinear system, however, terms beyond the quadratic can be neglected when evaluating the total energy. 
The damping time for solar $\mathrm{f}$ and low order g modes is about $10^{4}$ years. By this comparison, nonlinear damping would appear to be more rapid than linear damping if the energy of the primary is greater than $10^{34} \mathrm{erg}$, which is less than the minimum energy at which parametric instability can exist [eq. 13)]. So the nonlinear process should always dominate whenever it is allowed at all. But we shall revise this conclusion below.

We now consider the long-term evolution of the system. After a period $\sim t_{\mathrm{nl}}$, the energy in the primary mode drops by a factor $z 2$ and is shared among $N \sim \ell_{\max }^{3}(\Delta \omega / \omega) \sim \ell_{\max }^{3} / \ell_{\min }^{2}$ parametrically resonant daughter modes. Using eqs. (11)-(12) and the typical values of $\kappa_{\alpha \beta_{1} \beta_{2}}, t_{t h}$, and $\omega_{\alpha}$ cited above, we estimate $N \sim 10^{10} E_{0,42}^{5 / 4}$. The time scale for further energy decline is set by the daughter modes, which both dissipate energy by radiative process and also spawn lower frequency modes. The radiative damping time for g-modes of $\ell \sim 10^{4}$ is on order a week; and in fact this time is $\sim \eta^{-1}$ for modes of $\ell \sim \ell_{\max }$. Energy dissipated this way can clearly never return to global oscillations of the star.

Additionally, energy can also be removed from daughter modes if they themselves parametrically destabilize modes of still lower frequency - "granddaughter modes". The parametric growth rate of a granddaughter mode $(\gamma)$ due to nonlinearity in the daughter mode $(\beta)$ is $\eta^{\prime} \approx \omega_{\beta} \kappa_{\beta \gamma \gamma} A_{\beta}$. Energy transfer to the granddaughters will significantly retard the growth of the daughter modes when $\eta^{\prime} \geq \eta$, or in other words $\kappa_{\beta \gamma \gamma} A_{\beta} \geq \kappa_{\alpha \beta \beta} A_{0}$. Now it follows from an analysis of the 3-mode couplings that $\kappa_{\beta \gamma \gamma} \sim\left(\ell_{\beta} / \ell_{\alpha}\right) \kappa_{\alpha \beta \beta}$ if $\ell_{\beta} \gg \ell_{\alpha} \approx 2$ (Appendix A). Hence secondary parametric instabilities become important when $A_{\beta} \sim A_{0} / \ell_{\beta}$. Most of the daughter modes have degree $\ell_{\beta} \sim \ell_{\max }$, and after a time $\sim t_{\mathrm{nl}}$ each has a typical amplitude $A_{\beta} \sim N^{-1 / 2} A_{0} \sim\left(\ell_{\min }^{2} / \ell_{\max }^{3}\right)^{1 / 2} A_{0}$. This is smaller than $A_{0} / \ell_{\max }$ if $\ell_{\max }>\ell_{\min }^{2}$. From eqs. (11)-(12) we find $\ell_{\max } / \ell_{\min }^{2} \approx 25 E_{0,42}^{3 / 4}$.

Thus in the regime $E_{0} \ll 10^{40} \mathrm{erg}$, most of the daughter modes will be limited to energies much less than the equipartition value $\left(E_{0} / N\right)$ by secondary parametric instabilities. We can estimate how much this process should increase the nonlinear damping time of the primary mode. We assume that each daughter mode $(\beta)$ continues to absorb energy from the primary at a rate $\approx 2 \eta E_{\beta}$ even after $E_{\beta}$ has saturated. If saturation occurs at $E_{\beta} \sim\left(A_{0} / \ell_{\beta}\right)^{2}$, the primary loses energy at the rate

$$
\dot{E}_{\alpha} \sim-\eta \int_{\ell_{\min }}^{\ell_{\max }} \frac{E_{\alpha}}{\ell_{\beta}^{2}}\left(\frac{\ell_{\beta}}{\ell_{\min }}\right)^{2} d \ell_{\beta} \sim-\eta E_{\alpha}\left(\frac{\ell_{\max }}{\ell_{\min }^{2}}\right)
$$

instead of $\dot{E}_{\alpha}=-\eta E_{\alpha}$ as for $E_{\alpha} \gg 10^{40} \mathrm{erg}$. The modified nonlinear damping rate implied by eq. (21) scales as $E_{\alpha}^{5 / 4}$ instead of $E_{\alpha}^{1 / 2}$. Comparing this modified damping rate to the linear radiative damping of the primary mode $\left(\sim 10^{4} \mathrm{yr}\right)$, we find that nonlinear damping continues to dominate provided $E_{\alpha} \gtrsim 10^{37} \mathrm{erg}$, which is only an order of magnitude above the threshold for parametric instability $(\S 2.1)$.

At the energies expected of tidal capture $\left(E_{0} \sim 10^{42-45} \mathrm{erg}\right)$, the average energy per daughter mode is so small, because their number $N \propto E_{0}^{5 / 4}$ is so large, that the growth 
rate of the granddaughter modes can be neglected for the most part. However, secondary instabilities will prevent the fastest-growing daughters - those near the center of the instability strip ([6) - from exceeding an amplitude $\sim A_{0} / \ell_{\beta}$. By contrast, in the simplified model of eqs. (14)-(15) where secondary instabilities are ignored, the single fastest-growing mode briefly attains almost the entire energy of the system.

Low order g-modes of a radiative star carry a substantial amount of energy as a result of tidal interaction. The parametric instability described above will operate on these modes just as well, and independently of the f-modes, causing a loss of their energy on a time scale similar to the f-mode. This is discussed in more detail in $\S 4$.

\section{Nonlinear energy dissipation in convective stars}

The parametric nonlinear interaction mechanism described in the last section does not apply to fully convective stars, which do not have stable g-modes. However, even for these stars nonlinear mode coupling can be an important dissipation mechanism for the f-mode energy. The energy in f-modes of a convective star after tidal capture is often an order of magnitude larger compared to a radiative star of same mass and orbital parameters, and thus the nonlinear parameter, $A_{\alpha} \kappa_{\alpha \beta \gamma}$, is larger for convective stars. In this case even the normally very inefficient process of nonlinear coupling of a f-mode to higher frequency p-modes can transfer energy to short wavelength waves where it is dissipated on a relatively short time scale. The nonlinear response to the f-mode can be treated as a forced excitation of all p-modes of degree $\ell=0,2$, and 4 , in which the forcing function is quadratic in the f-mode amplitude and oscillates at twice the f-mode frequency. We calculate this response by solving an inhomogenous wave equation for the sum of the p-mode amplitudes with the nonlinear forcing function as the inhomogenous term. The latter can be obtained either from the Lagrangian given in Appendix A, or by expanding mass, momentum and energy equations to second order in wave quantities [e.g. Dzimbowski (1982)]. The solution of the inhomogeneous equation is shown in figure 2 together with the f-mode eigenfunction. The turbulent viscous, and thermal, dissipation of wave energy is calculated using this wavefunction. Note the generation of significant power at short wavelengths and the relatively large displacement amplitude and its derivative in the outer part of the star; these effects enhance the dissipation of f-mode energy. The resulting nonlinear dissipation time for the f-mode by coupling to p-modes is

$$
t_{f f p}=3 \times 10^{4} E_{42}^{-1} \text { days, }
$$

where $E_{42}$ is f-mode energy in units of $10^{42} \mathrm{erg}$.

We can estimate the damping rate in another way that is less accurate but provides some insight. In the present special case of the general 3-mode interaction (1), we drop all 
nonlinear terms except the square of the f-mode amplitude. The result is

$$
\ddot{A}_{\beta}+\omega_{\beta}^{2} A_{\beta}=-3 \omega_{\beta}^{2} \kappa_{\beta \alpha \alpha} A_{\alpha}^{2}
$$

where $A_{\alpha}$ is the amplitude of the f-mode which we take to be $A_{0} \sin \left(\omega_{\alpha} t\right)$. Substituting this into the above equation we find for the amplitude of mode $\beta$,

$$
A_{\beta}=-\frac{3 \omega_{\beta}^{2} A_{0}^{2} \kappa_{\beta \alpha \alpha} \cos \left(2 \omega_{\alpha} t\right)}{2\left(\omega_{\beta}^{2}-4 \omega_{\alpha}^{2}\right)} .
$$

The net displacement associated with the p-mode amplitudes $A_{\beta}$ is

$$
\vec{\xi}=\sum_{\beta} A_{\beta} \vec{\xi}_{\beta}
$$

which can be used to calculate the dampings. The function $A_{\beta} \vec{\xi}_{\beta}$ has alternating signs for even and odd order modes near the surface of the star where most of the damping is taking place. This can lead to significant cancellation in the total displacement. Nevertheless we proceed by assuming that different modes can be added in quadrature. The energy in mode $\beta$ is

$$
\left\langle E_{\beta}\right\rangle=\frac{9}{16} \frac{\omega_{\beta}^{4}\left(A_{0} \kappa_{\beta \alpha \alpha}\right)^{2} A_{0}^{2}}{\left(\omega_{\beta}^{2}-4 \omega_{\alpha}^{2}\right)^{2}}\left[1+\frac{4 \omega_{\alpha}^{2}}{\omega_{\beta}^{2}}\right] .
$$

If the rate of dissipation of energy of a mode $\beta$ driven at frequency $2 \omega_{\alpha}$ is $\Gamma_{\beta}$, then the rate of energy dissipation of the f-mode (mode $\alpha$ ) can be calculated from this last equation, and the resulting dissipation time is

$$
t_{f f p} \sim\left(A_{0}^{2} \sum_{\beta} \Gamma_{\beta} \kappa_{\beta \alpha \alpha}^{2}\right)^{-1} .
$$

The 3 -mode coupling coefficients, $\kappa_{f f p}$, involving a quadrupole $\mathrm{f}$ and a radial $\mathrm{p}$ mode for a solar model, have a typical value $10^{-24} \mathrm{erg}^{-1 / 2}$, and the damping time for high order p-modes $\left(1 / \Gamma_{p}\right)$ is about 10 days. Since there are about 100 p-modes of degree 0 , 2, and 4 coupled to the quadrupole f-mode, we see from equation (27) that for a f-mode of energy $10^{42} \mathrm{erg}$, the non-linear damping time is about $10^{4}$ days which is within a factor of a few of the numerically calculated value given in eq. (22). For comparison we note that the linear turbulent viscous damping time of f-mode is about $10^{4}$ years.

The energy in the f-mode of a 0.5 solar mass polytropic star of index 1.5, tidally captured by a one solar mass star, is about $10^{45} \operatorname{erg}\left(\delta R / R_{*} \approx 0.1\right)$, and so its nonlinear dissipation [22)] time is about 30 days. At such high energies, however, the system is very nonlinear: that is, four mode couplings are as important as the three mode couplings.

Figure 2 shows that for this energy the amplitude of the p-mode response is about the same (or greater) as the f-mode amplitude throughout the star, and thus higher order couplings must be equally important. In this case it is very likely that high order p-modes are 
resonantly excited leading to even more efficient dissipation to f-mode energy. Moreover, since most of the energy is dissipated in the outermost envelope of the star at a rate of $\sim 10^{39} \mathrm{erg} \mathrm{s}^{-1}$, which is more than can be transported by convection, it will cause the envelope to expand away from the star. This is a further indication of the breakdown of the weak nonlinear calculation presented here.

In addition to the nonlinear effect arising from the third order fluid Lagrangian, considered here, there is also nonlinearity associated with the thermal dissipation process itself. It can be shown that both of these are of the same order, and thus a neglect of the latter does not change our result by more than a factor of a few, which is within the uncertainty of the calculation of this section.

\section{Application to capture binaries in globular clusters}

Main sequence stars of mass greater than $0.5 M_{\odot}$ have a sizable radiative core (see table 2) that supports a dense family of g-modes. When such stars are tidally captured, resulting in a highly eccentric orbit with separation between the stars at periastron of only a few stellar radii initially, their quadrupole $f$ and low order g modes are excited to large amplitudes. The linear radiative damping time for these modes is of order $10^{4}$ years. However these modes are resonantly coupled to high degree g-modes by parametric instability which provides a very efficient way of dissipating their energy (see §2). We now estimate the time for the energy of the low order modes to decay due to nonlinear interaction using the results of $\S 2$.

The main ingradient required for nonlinear dissipation calculation is the 3-mode coupling coefficients, $\kappa_{\alpha \beta_{1} \beta_{2}}$, which are computed using equation (A17). The numerical calculation of $\kappa_{\alpha \beta_{1} \beta_{2}}$ is carried out for a simplified polytropic stellar model, which is widely used in tidal capture and evolution calculations, as well as a standard solar model. The main sequence globular cluster stars of mass greater than about $0.5 M_{\odot}$ have a radiative core (see table 2) and can be crudely modelled as a polytrope of index 3 [cf. Pinsonneault, Deliyannis, \& Demarque (1992), Lai, Rasio, \& Shapiro (1994), Deliyannis (1995)]. The coupling coefficients involving low degree g-modes $\left(\ell_{\beta} \leq 40\right)$ are calculated using eigenfunctions obtained by solving the wave equation, whereas for high degree g-modes we use the WKB solution which is an excellent approximation. We find that $\kappa_{\alpha \beta \beta}$ for the Sun and $1.0 M_{\odot}$ polytropic star of index 3 agree to within a factor of two for $\ell_{\beta} \lesssim 100$ (the polytropic model yields a higher value). However, coupling coefficients increase with $\ell_{\beta}$ for polytropic models but not in the case of the Sun or a realistic stellar model. This is because g-modes are trapped in the radiative interior of low mass main sequence stars, whereas in a polytropic model, where the Brunt-Väisälä frequency increases monotonically with distance from the center, g-modes propagate closer and closer to the surface with increasing mode degree. One should therefore use a realistic stellar model 
for the purpose of nonlinear mode interaction calculation. Since $\kappa_{\alpha \beta_{1} \beta_{2}} \sim G^{-1 / 2} R_{*}^{1 / 2} M_{*}^{-1}$ depends only weakly on stellar mass we use a solar model to compute coupling coefficients that are used for lower mass globular cluster stars; the true coupling coefficients are larger by about $20 \%$. Table 1 shows the value of $\kappa_{\alpha \beta \beta}$ for a selected number of modes. Note that a typical value of coupling coefficient is $10^{-24} \mathrm{erg}^{-1 / 2}$ which is in good agreement with the rough estimate of $G^{-1 / 2} R_{*}^{1 / 2} M_{*}^{-1}$.

The dependence of $\kappa_{\alpha \beta \beta}$ on $m_{\beta}$ is entirely contained in Wigner 3-J symbol [see eq. (A10)], which for large $\ell_{\beta}$ scales as $\cos \left(\pi m_{\beta} / 2 \ell_{\beta}\right)$. Thus a good fraction of modes of all possible $m$, for a given $\ell$, are equally well coupled to the $f$ and low order g modes. Finally, we note that the coupling coefficient decreases with increasing $\Delta n \equiv\left|n_{\beta_{1}}-n_{\beta_{2}}\right|$ and is neglegible if $\Delta n \gtrsim 10$.

The energy input rate per orbital period, for several different modes, are shown in figure 3 as a function of distance between the stars at periastron. This was calculated using a scheme similar to Press \& Teukolsky (1977). In general most of the energy is in some g-mode which has a period close to the periastron passage time. Substituting mode amplitudes and coupling coefficients into equation (19) we obtain nonlinear damping times which are displayed in figure 4 for several modes as a function of periastron separation. Note that for mode energy of $10^{42} \mathrm{erg}$ (corresponding to dimensionless surface displacement amplitude $\delta R / R_{*} \approx 2 \times 10^{-3}$; see Table 1 ) the nonlinear damping time is 50 days. During this time the mode energy decreases by a large factor, which is shared equally by about $N \sim 10^{10}$ high degree g-modes that have been parametrically excited via nonlinear mode coupling. It follows from equation (19) that the nonlinear damping time scales as $E_{\alpha}^{-1 / 2}$, and thus for mode energy of $10^{44} \mathrm{erg}$ the damping time is only 5 days. For comparison we note that the radiative damping time for low order quadrupole modes are of order $10^{4}$ years. Thus nonlinear dissipation dominates even when periastron distance is $4 R_{*}$ (see fig. 3 \& 4 ). In fact the nonlinear dissipation rate is larger than the linear radiative damping so long as mode energy is greater than about $10^{37} \mathrm{erg}$. It should be emphasized that energy removed from a mode by nonlinear interactions is distributed over a large number of g-modes. The energy is eventually thermalized on the dissipation time scale of high degree g-modes, which for modes of $\ell \sim \ell_{\max }$ is of the same order as $t_{\mathrm{nl}}$. How the star adjusts to the energy deposited in its core on a short time scale is outside of the scope of this work. However, we note that even $10^{44} \mathrm{erg}$ of energy deposited in the radiative core is a small fraction of its thermal energy content, and thus will have a minor effect on the structure of the star.

Main sequence globular cluster stars of mass less than about $0.5 M_{\odot}$ are almost fully convective, and therefore the coupling of f-mode to g-modes is very weak. The dominant nonlinear coupling of the f-mode in this case, as described in $\S 3$, is with p-modes. Physically this nonresonant mode coupling results in power on smaller length scale, and also the value of the gradient of the wave function generated can be considerably larger than the original f-mode wave function in the outer part of the star (see fig. 2). This leads to more efficient dissipation of wave energy. As discussed in $\S 3$ the f-mode dissipation time due to this process is about $3 \times 10^{4} \mathrm{E}_{42}^{-1}$ days. 


\section{Summary}

Tidally excited modes of a main sequence star in a highly eccentric orbit, with periastron distance of 4 stellar radii or less, have energy about $10^{42}$ erg or greater, or fractional change in stellar radius of $0.2 \%$ or more. The linear radiative and turbulent dissipation times for these modes are about $10^{4}$ years. We have calculated the time needed for energy of tidally excited modes to decrease due to nonlinear interaction with other modes in the star for two different cases that are described below.

One of the cases considered is that of a star with a radiative core - for instance main sequence globular cluster stars of mass greater than about $0.5 \mathrm{M}_{\odot}$. The most important mode coupling in these radiative stars involves high degree g-modes which are parametrically excited by the low order quadrupole modes. The time scale for energy

transfer for this process is $2 /\left(\nu_{\alpha} \kappa_{\alpha \beta \beta} E_{\alpha}^{1 / 2}\right)$, where $\kappa_{\alpha \beta \beta} \sim G^{-1 / 2} M_{*}^{-1} R_{*}^{1 / 2} \sim 10^{-24} \mathrm{erg}^{-1 / 2}$ is 3-mode coupling coefficient. Thus for $E_{\alpha} \approx 10^{42} \mathrm{erg}$, or $\delta R / R_{*} \approx 2 \times 10^{-3}$, the nonlinear damping time is 50 days. This is smaller by a factor of $10^{6}$ compared to the nonlinear damping time estimated by Kochanek (1992), who had not considered parametric coupling. The energy removed from the primary mode initially resides in roughly $10^{10}$ high degree g-modes and is later dissipated in the radiative core of the star on a time scale of about a year. Since this energy is small compared to the thermal energy content of the core, it is unlikely to have much effect on the structure of the star in one orbital passage.

The other case we have considered is that of a fully convective star, such as a main sequence star of mass less than about $0.5 \mathrm{M}_{\odot}$. Most of the tidal energy in these stars, which do not have stable g-modes, resides in quadrupole f-modes. The f-modes couple to high order p-modes of degrees $\ell=0,2$, and 4 generating short wavelength waves that significantly enhance the energy dissipation rate. The energy of short wavelength p-modes is dissipated near the stellar surface by turbulent and radiative viscosity. The nonlinear energy dissipation time of $\mathrm{f}$-modes is estimated to be $3 \times 10^{4}\left(10^{42} \mathrm{erg} / E_{f}\right)$ days or $2 \times 10^{-1}$ $\left(\delta R / R_{*}\right)^{-2}$ days, which is shorter than the linear dissipation time when the mode energy is greater than about $10^{40} \mathrm{erg}$. And it is also smaller than the nonlinear damping time estimate of Kochanek (1992) by a factor of about $10^{3}$. For mode energy of $10^{45}$ erg, that which is expected in tidal captures, the nonlinear damping time is about 30 days. However, for such a large energy, 4-mode 5-mode, and higher-order mode coupling are also important; that is, the weak nonlinear approximation is not valid.

Acknowledgment: PK is grateful to Piet Hut for suggesting this problem, and he is indebted to John Bahcall for his support and hospitality at the IAS where this work was carried out. We thank Peter Goldreich, Piet Hut, Steve Lubow, John Papaloizou, and J.E. Pringle for useful discussions - the last three in particular for prompting us to add Appendix C. JG acknowledges NASA support under grant NAG5-2796. 


\section{A. Adiabatic g-mode couplings in spherical geometry}

The Lagrangian for inviscid, compressible fluid with internal energy $E(s, \rho)$ is

$$
\mathcal{L}=\rho\left(\frac{v^{2}}{2}-E(s, \rho)-U(\mathbf{x})\right)
$$

where $v$ is fluid velocity, $s, \rho$, and $U$ are entropy, density, and gravitational potential. The three mode coupling is described by the fluid Lagrangian expanded to third order in displacement and is given below

$$
\begin{aligned}
\mathcal{L}_{3} & =-\frac{1}{2} \xi^{i} \xi^{j} \delta p_{; i ; j}-\delta p_{; i} \xi^{i} \vec{\nabla} \cdot \vec{\xi}-\frac{1}{2}(\vec{\nabla} \cdot \vec{\xi})^{2} \delta p \\
& +\frac{\gamma(\gamma-2)}{6} p(\vec{\nabla} \cdot \vec{\xi})^{3}-\frac{1}{2} p_{; i} \xi^{i}(\vec{\nabla} \cdot \vec{\xi})^{2}-\frac{1}{2} p_{; i ; j} \xi^{i} \xi^{j} \vec{\nabla} \cdot \vec{\xi} \\
& -\frac{1}{6} p_{; i ; j ; k} \xi^{i} \xi^{j} \xi^{k}-\frac{1}{6} U_{; i ; j ; k} \rho \xi^{i} \xi^{j} \xi^{k}-\frac{1}{2} \rho \xi^{i} \xi^{j} \delta U_{; i ; j} .
\end{aligned}
$$

Here $p(r)$ is the equilibrium pressure profile, $\xi$ is the fluid displacement from its equilibrium position, semicolon denotes covariant derivative (see Misner, Thorne, and Wheeler 1973 for the definition of covariant derivative), and $\delta p$ is the Eulerian pressure perturbation to first order in $\xi$ :

$$
\delta p \equiv-\vec{\xi} \cdot \vec{\nabla} p-\frac{\gamma p}{\rho} \vec{\nabla} \cdot \vec{\xi}
$$

Let $\xi \rightarrow \xi+\Xi$, where $\Xi$ is a large-scale fundamental or g-mode and $\xi$ becomes the sum of all small-scale modes. Then the dominant (i.e., leading order in $\ell$ of small-scale modes) terms are

$$
\begin{aligned}
\mathcal{L}_{3} & =\Xi_{; i}^{j} \xi^{i} \delta p_{; j}-\frac{1}{2} \xi^{i} \xi^{j}\left(\delta P_{; i ; j}+\rho \delta U_{; i ; j}\right)-\vec{\nabla} \cdot \vec{\xi} \xi^{i} \delta P_{; i}-\frac{1}{2}(\vec{\nabla} \cdot \vec{\xi})^{2} \delta P \\
& +\frac{\gamma(\gamma-2)}{2} p(\vec{\nabla} \cdot \vec{\xi})^{2} \vec{\nabla} \cdot \vec{\Xi}-\frac{1}{2} p_{; i} \Xi^{i}(\vec{\nabla} \cdot \vec{\xi})^{2}-p_{; i} \xi^{i} \vec{\nabla} \cdot \vec{\xi} \vec{\nabla} \cdot \vec{\Xi} \\
& -p_{; i ; j}\left(\frac{1}{2} \xi^{i} \xi^{j} \vec{\nabla} \cdot \vec{\Xi}+\xi^{i} \Xi^{j} \vec{\nabla} \cdot \vec{\xi}\right)-\frac{1}{2}\left(p_{; i ; j ; k}+\rho U_{; i ; j ; k}\right) \xi^{i} \xi^{j} \Xi^{k}
\end{aligned}
$$

Capital letters are used to distinguish quantities pertaining to the large-scale mode: e.g. $\delta P$ and $\delta U$ are the first-order eulerian pressure and potential perturbations associated with $\Xi$. (However $U$ unadorned with $\delta$ is the equilibrium potential.) The 3-mode coupling coefficient is the integral of $\mathcal{L}_{3}$ over the star, when the mode eigenfunctions are normalized to unit energy.

Integration by parts has been used in deriving formulae (A2) and (A4) to remove as

many derivatives as possible from the small-scale displacements $\vec{\xi}$, except where these occur 
as divergences $\vec{\nabla} \cdot \vec{\xi}$. In general $\xi_{; j}^{i} \sim \xi / \lambda$, where $\lambda \ll R_{*}$ is lengthscale associated with the displacement. Because the small-scale g-modes are nearly incompressible and isobaric, however, $\vec{\nabla} \cdot \vec{\xi} \sim \xi / H$ and $\delta p / p \sim \xi \lambda / H^{2}$, where $H \sim R_{*}$ is the pressure or density scale height.

The next step is to integrate $\mathcal{L}_{3}$ over solid angle $\left(d^{2} \Omega=\sin \theta d \theta d \phi\right)$. As usual, the covariant components of the angular displacements can be written as partial derivatives of a scalar potential [here and henceforth, greek indices range over the angular coordinates $\{\theta, \phi\}$, whereas roman ones range over $\{r, \theta, \phi\}]$,

$$
\xi_{\alpha}=\partial_{\alpha} \psi, \quad \Xi_{\alpha}=\partial_{\alpha} \Psi
$$

and the displacements can be decomposed into angular harmonics:

$$
\begin{aligned}
\xi^{r}=\sum_{\Lambda} \xi_{\Lambda}^{r}(t, r) Y_{\Lambda}(\theta, \phi), & \psi=\sum_{\Lambda} \psi_{\Lambda}(t, r) Y_{\Lambda}(\theta, \phi), \\
\Xi^{r}=\sum_{\Lambda_{f}} \Xi_{\Lambda_{f}}^{r}(t, r) Y_{\Lambda_{f}}(\theta, \phi), & \Psi=\sum_{\Lambda_{f}} \Psi_{\Lambda_{f}}(t, r) Y_{\Lambda_{f}}(\theta, \phi) .
\end{aligned}
$$

We use the single symbol $\Lambda$ for the degree and order $(\ell, m)$ of a spherical harmonic i.e., $Y_{\Lambda} \equiv Y_{\ell m}(\theta, \phi)$, and define $\Lambda^{2} \equiv \ell(\ell+1)$. The projection of a function $f(t, r, \theta, \phi)$ onto $Y_{\ell m}$ is written $f_{\Lambda}$ and is understood to be a function of $(t, r)$ (except that $Y_{\Lambda}$ denotes $Y_{\ell m}(\theta, \phi)$ ):

$$
f_{\Lambda} \equiv \int f(t, r, \theta, \phi) Y_{\Lambda}^{*}(\theta, \phi) d^{2} \Omega
$$

Enclosing a term in angle brackets means to integrate it over solid angle; thus the above could be written $f_{\Lambda}=\left\langle Y_{\Lambda}^{*} f\right\rangle$. The angular integral of three spherical harmonics is related to the Wigner 3j symbol (see Edmonds, 1960),

$$
\left(\begin{array}{ccc}
\ell_{1} & \ell_{2} & \ell_{3} \\
m_{1} & m_{2} & m_{3}
\end{array}\right)
$$

by

$$
\begin{aligned}
\left\langle\Lambda_{1} \Lambda_{2} \Lambda_{3}\right\rangle & \equiv\left\langle Y_{\Lambda_{1}} Y_{\Lambda_{2}} Y_{\Lambda_{3}}\right\rangle \\
& =\left[\frac{\left(2 \ell_{1}+1\right)\left(2 \ell_{2}+1\right)\left(2 \ell_{3}+1\right)}{4 \pi}\right]^{1 / 2}\left(\begin{array}{ccc}
\ell_{1} & \ell_{2} & \ell_{3} \\
m_{1} & m_{2} & m_{3}
\end{array}\right)\left(\begin{array}{ccc}
\ell_{1} & \ell_{2} & \ell_{3} \\
0 & 0 & 0
\end{array}\right) .(A)
\end{aligned}
$$

To calculate $\left\langle\mathcal{L}_{3}\right\rangle$ from eq. (A4), one needs the following identities:

$$
\begin{gathered}
\left\langle Y_{\Lambda_{3}} \hat{g}^{\alpha \beta}\left(\hat{\nabla}_{\alpha} Y_{\Lambda_{1}}\right)\left(\hat{\nabla}_{\beta} Y_{\Lambda_{2}}\right)\right\rangle=\frac{\Lambda_{1}^{2}+\Lambda_{2}^{2}-\Lambda_{3}^{2}}{2}\left\langle\Lambda_{1} \Lambda_{2} \Lambda_{3}\right\rangle, \\
\left\langle\hat{g}^{\mu \alpha} \hat{g}^{\nu \beta}\left(\hat{\nabla}_{\mu} \hat{\nabla}_{\nu} Y_{\Lambda_{3}}\right)\left(\hat{\nabla}_{\alpha} Y_{\Lambda_{1}}\right)\left(\hat{\nabla}_{\beta} Y_{\Lambda_{2}}\right)\right\rangle=\frac{\left(\Lambda_{3}^{2}\right)^{2}-\left(\Lambda_{1}^{2}-\Lambda_{2}^{2}\right)^{2}}{4}\left\langle\Lambda_{1} \Lambda_{2} \Lambda_{3}\right\rangle .
\end{gathered}
$$


Here $\hat{\nabla}$ is the usual covariant derivative on the sphere; that is, $\hat{\nabla}_{\gamma} \hat{g}_{\alpha \beta}=0$, where $\hat{g}_{\alpha \beta}$ is the 2D metric

$$
\hat{g}_{\alpha \beta} d x^{\alpha} d x^{\beta}=d \theta^{2}+\sin ^{2} \theta d \phi^{2}
$$

and $\hat{g}^{\alpha \beta}$ is its inverse.

To establish Eq. (A11), for example, note that

$$
\hat{g}^{\alpha \beta}\left(\hat{\nabla}_{\alpha} Y_{\Lambda_{1}}\right)\left(\hat{\nabla}_{\beta} Y_{\Lambda_{2}}\right)=\frac{1}{2}\left[\hat{\nabla}^{2}\left(Y_{\Lambda_{1}} Y_{\Lambda_{2}}\right)-Y_{\Lambda_{1}} \hat{\nabla}^{2} Y_{\Lambda_{2}}-\left(\hat{\nabla}^{2} Y_{\Lambda_{1}}\right) Y_{\Lambda_{2}}\right]
$$

and that

$$
\hat{\nabla}^{2} Y_{\Lambda} \equiv \hat{g}^{\alpha \beta} \hat{\nabla}_{\alpha} \hat{\nabla}_{\beta} Y_{\Lambda}=\Lambda^{2} Y_{\Lambda}
$$

and use integration by parts. The $2 \mathrm{D}$ covariant derivative is not simply the restriction of the $3 \mathrm{D}$ covariant derivative to the sphere, but is related to it by formulae such as

$$
\begin{aligned}
f_{; \alpha} & =\hat{\nabla}_{\alpha} f=\partial_{\alpha} f \\
v_{; \alpha}^{r} & =\hat{\nabla}_{\alpha} v^{r}-r^{-1} v_{\alpha}, \partial_{\alpha} v^{r}-r^{-1} v_{\alpha}, \\
v_{\alpha ; \beta} & =\hat{\nabla}_{\beta} v_{\alpha}-r \hat{g}_{\alpha \beta} v_{r},
\end{aligned}
$$

where $f$ and $v^{i}$ are scalar and vector fields in 3D. To avoid confusion, we raise and lower indices using $g_{i j}$ only: for example, $v_{\alpha} \equiv g_{\alpha i} v^{i}\left(\right.$ not $\left.\hat{g}_{\alpha \beta} v^{\beta}\right)$.

Using these rules we integrate the third order Lagrangian over angle which leads to the following expression

$$
\begin{aligned}
& \int \mathcal{L}_{3} d^{2} \Omega=\sum_{\Lambda_{1}, \Lambda_{2}, \Lambda_{f}}\left\langle\Lambda_{1} \Lambda_{2} \Lambda_{f}\right\rangle\{ \\
& \frac{\left(\Lambda_{f}^{2}\right)^{2}-\left(\Lambda_{1}^{2}-\Lambda_{2}^{2}\right)^{2}}{4 r^{4}}\left[\Psi_{\Lambda_{f}} \psi_{\Lambda_{1}} \delta p_{\Lambda_{2}}-\frac{1}{2}(\delta P+\rho \delta U)_{\Lambda_{f}} \psi_{\Lambda_{1}} \psi_{\Lambda_{2}}\right]+ \\
& \frac{\Lambda_{1}^{2}+\Lambda_{2}^{2}-\Lambda_{f}^{2}}{2 r^{2}}\left[r \Xi_{\Lambda_{f}}^{r} \psi_{\Lambda_{1}} \delta p_{\Lambda_{2}}-\frac{1}{2}\left(r \partial_{r} \delta P+\rho r \partial_{r} \delta U+\frac{1}{r} \frac{d p}{d r} \vec{\nabla} \cdot \vec{\Xi}\right.\right. \\
& \left.\left.+\frac{d}{d r}\left(\frac{1}{r} \frac{d p}{d r}\right) \Xi^{r}\right)_{\Lambda_{f}} \psi_{\Lambda_{1}} \psi_{\Lambda_{2}}\right]+ \\
& \frac{\Lambda_{f}^{2}+\Lambda_{1}^{2}-\Lambda_{2}^{2}}{2 r^{2}}\left[\left(\Xi^{r}-\frac{1}{r} \Psi\right)_{\Lambda_{f}} \psi_{\Lambda_{1}} \partial_{r} \delta p_{\Lambda_{2}}+r \partial_{r}\left(\frac{1}{r} \Psi\right)_{\Lambda_{f}} \delta p_{\Lambda_{1}} \xi_{\Lambda_{2}}^{r}-r \partial_{r}\left(\frac{1}{r} \delta P\right)_{\Lambda_{f}} \psi_{\Lambda_{1}} \xi_{\Lambda_{2}}^{r}\right. \\
& \left.-\rho r \partial_{r}\left(r^{-1} \delta U\right)_{\Lambda_{f}} \psi_{\Lambda_{1}} \xi_{\Lambda_{2}}^{r}-\left(\delta P+\frac{1}{r} \frac{d p}{d r} \Psi\right)_{\Lambda_{f}} \psi_{\Lambda_{1}} \vec{\nabla} \cdot \vec{\xi}_{\Lambda_{2}}-\frac{d}{d r}\left(\frac{1}{r} \frac{d p}{d r}\right) \Psi_{\Lambda_{f}} \psi_{\Lambda_{1}} \xi_{\Lambda_{2}}^{r}\right]+ \\
& {\left[\partial_{r} \Xi_{\Lambda_{f}}^{r} \xi_{\Lambda_{1}} \partial_{r} \delta p_{\Lambda_{2}}-\left(\partial_{r} \delta P+\frac{d p}{d r} \vec{\nabla} \cdot \vec{\Xi}+\frac{d^{2} p}{d r^{2}} \Xi^{r}\right)_{\Lambda_{f}} \xi_{\Lambda_{1}}^{r} \vec{\nabla} \cdot \vec{\xi}_{\Lambda_{2}}\right.}
\end{aligned}
$$




$$
\left.\begin{array}{l}
-\frac{1}{2}\left(\delta P+\gamma(\gamma-2) p \vec{\nabla} \cdot \vec{\Xi}+\frac{d p}{d r} \Xi^{r}\right)_{\Lambda_{f}} \vec{\nabla} \cdot \vec{\xi}_{\Lambda_{1}} \vec{\nabla} \cdot \vec{\xi}_{\Lambda_{2}} \\
\left.-\frac{1}{2}\left(\frac{d^{2} p}{d r^{2}} \vec{\nabla} \cdot \vec{\Xi}+\frac{d^{3} p}{d r^{3}} \Xi^{r}+\rho \frac{d^{3} U}{d r^{3}} \Xi^{r}+\rho \partial_{r}^{2} \delta U+\partial_{r}^{2} \delta P\right)_{\Lambda_{f}} \xi_{\Lambda_{1}}^{r} \xi_{\Lambda_{2}}^{r}\right]
\end{array}\right\}
$$

Every term in this expression is formally of order $\left(\xi^{r} / H\right)^{2} p$, where $\xi^{r}=\xi_{\Lambda_{1}}^{r}$ or $\xi_{\Lambda_{2}}^{r}$, since $\psi_{\Lambda_{1}} \sim R \xi_{\Lambda_{1}}^{r} / \ell$. The 3-mode coupling coefficent, $\kappa_{\alpha \beta \gamma}$, is obtained by integrating the above expression over the radius of the star i.e.

$$
\kappa_{\alpha \beta \gamma}=\int d r r^{2} \int d^{2} \Omega \mathcal{L}_{3}
$$

Note that all the eigenfuctions in equation (A17) are normalized to unit energy.

When all three interacting modes have wavelengths $\left(\lambda_{1}, \lambda_{2}, \lambda_{3}\right) \ll\left(R_{*}, H\right)$, then the first term on the righthand side of (A2) dominates the rest by a factor $\sim H / \max \left(\lambda_{1}, \lambda_{2}, \lambda_{3}\right)$. This justifies our statement in the text that the coefficient $\kappa_{\beta \gamma \gamma}$ coupling daughter to granddaughter modes exceeds the coupling $\kappa_{\alpha \beta \beta}$ of the primary mode to its daughters by a factor $\sim \ell_{\beta}$. Physically this can be understood, or at least remembered, by noting that the parametric growth rate scales as the shear $\sim \omega \xi / \lambda$ in the parent mode. 


\section{B. Rotational splitting of eigenfrequencies}

The rotational splitting of mode frequencies as seen in the mean rotational frame of the star is given by (e.g. Stix (1991))

$$
\Delta \nu_{n \ell m} \equiv \nu_{n \ell m}-\nu_{n \ell 0}=-m C_{n \ell} \Omega_{0} / 2 \pi
$$

where $\Omega_{0}$ is the mean angular speed of the star,

$$
C_{n \ell}=\frac{\int d^{3} x \rho\left[\xi_{h}^{2}+2 \xi_{r} \xi_{h}-\left(\Delta \Omega / \Omega_{0}\right)\left\{\left(\xi_{r}-\xi_{h}\right)^{2}+\left(\ell^{2}+\ell-2\right) \xi_{h}^{2}\right\}\right]}{\int d^{3} x \rho\left[\xi_{r}^{2}+\ell(\ell+1) \xi_{h}^{2}\right]},
$$

and

$$
\Delta \Omega(r) \equiv \Omega(r)-\Omega_{0} .
$$

For high order g-modes $\xi_{r} \sim i \ell \xi_{h}$, and thus $\int d^{3} x \rho \xi_{r} \xi_{h} \approx 0$. Substituting these into equation (B2) we find that for a rigidly rotating star

$$
C_{n \ell} \approx \frac{1}{2 \ell^{2}}
$$

and so the frequency difference between modes of $m=\ell$ and $-\ell$ is $\Omega_{0} / 2 \pi \ell$. For a differentially rotating star the splitting coefficient is

$$
C_{n \ell} \approx \frac{1}{2 \ell^{2}}-\frac{\overline{\Delta \Omega}}{\Omega_{0}}
$$

where $\overline{\Delta \Omega}$ is the mean value of differential rotation in the radiative interior of star where high order g-modes are trapped. This leads to $\Delta \nu_{n \ell m} \approx-m \overline{\Delta \Omega} / 2 \pi$ if $\ell \gg 1$. However, if this formula predicts $\Delta \nu_{n \ell m}>N_{B}$, the Brunt-Väisälä frequency, then individual g-modes are trapped within regions smaller than the entire radiative zone, and the spectrum of mode frequencies becomes continuous. 


\section{Frequency shifts and saturation}

Mechanical oscillations and waves are generally anharmonic: their natural frequency depends upon amplitude. Unless other processes intervene earlier, a parametric instability will saturate at an amplitude such that the resonance condition (6) is no longer satisfied because of the anharmonic frequency shift.

For example, a generic one-dimensional oscillator with Hamiltonian

$$
H=\frac{1}{2 m} p^{2}+m \omega_{0}^{2} q^{2}\left\{\frac{1}{2}+\frac{\kappa}{3 a_{0}} q+\frac{\mu}{4 a_{0}^{2}} q^{2}+O\left[\left(q / a_{0}\right)^{3}\right]\right\}
$$

has free oscillations (cf. Landau \& Lifshitz 1976)

$$
\begin{aligned}
q(t) & =a \cos [\omega(a) t] \\
\omega(a) & =\omega_{0}\left[1+\left(\frac{3}{8} \mu-\frac{5}{12} \kappa^{2}\right)\left(\frac{a}{a_{0}}\right)^{2}+O\left(\frac{a^{4}}{a_{0}^{4}}\right)\right] .
\end{aligned}
$$

Here $a_{0}$, a characteristic amplitude at which anharmonicity becomes important, is chosen so that the larger of $\mu$ and $\kappa^{2}$ is of order unity. Suppose this oscillator is coupled to another in such a way that very small oscillations are parametrically unstable with growth rate $\eta=\epsilon \omega_{0}$. The range of frequencies over which parametric instability occurs is $\Delta \omega \approx \eta$. If (for simplicity) parametric resonance is exact at infinitesimal amplitude, then growth stops when $\left|\omega(a)-\omega_{0}\right| \approx \eta$, hence $a \sim \epsilon^{1 / 2} a_{0}$.

Waves in fluids are normally strongly nonlinear when $k a \sim 1$, where $a=\max (|\vec{\xi}|)$ is the peak displacement and $k=2 \pi / \lambda$ is the wavenumber. That is, we may normally take $a_{0}=\lambda / 2 \pi$. This is because the eulerian dynamical equations contain the advective derivative $\partial_{t}+\vec{v} \cdot \vec{\nabla}$, and for small perturbations of a static equilibrium, the ratio of the second (nonlinear) part of this operator to the first is $O(k \delta v / \omega)=O(k a)$. A single $\mathrm{g}$ mode, however, is an exception to this general rule if the wavelength is small compared to a pressure or density scale height, $H$. Such a wave is nearly incompressible, so that if $\delta \vec{v} \propto \exp (i \vec{k} \cdot \vec{x})$ in a local plane-wave approximation, then $\vec{\nabla} \cdot \delta \vec{v}$ is $\sim \delta v / H$ instead of $\sim k \delta v$. It follows that the operator $\vec{v} \cdot \vec{\nabla}=O(\delta v / H)$ when applied to any fluid perturbation whose WKBJ wavenumber is parallel to that of $\delta \vec{v}$. One can check that other nonlinear terms vanish to leading order in $(k H)^{-1}$. For example, the dynamical equation for the vorticity $\vec{\nabla} \times \vec{v}$ contains the source term $\rho^{-2}(\vec{\nabla} \rho \times \vec{\nabla} p)$; but for a single wave, the gradients of the density and pressure perturbations are both approximately parallel to the wavenumber, so that $\vec{\nabla} \delta \rho \times \vec{\nabla} \delta p$ vanishes to leading order in $(k H)^{-1}$.

We note in passing that nonlinear interactions involving two or more nonparallel wavenumbers need not be similarly suppressed. In parametric instability, for example, the primary and daughter modes have nonparallel wavenumbers. Anharmonicity is a special nonlinearity because it involves self-interaction of a single mode. 
Consequently, the anharmonic frequency shift of a WKB g mode is

$$
\omega(a)-\omega_{0}=O\left(\omega_{0} \frac{a^{2}}{H^{2}}\right) .
$$

Notice that this does not depend on mode wavelength. Equating this shift to the width $\Delta \omega \sim \epsilon \omega_{0}$ of the parametric resonance caused by a fundamental mode of dimensionless amplitude $\epsilon=\delta R_{*} / R_{*}$, one predicts saturation when the small-scale mode has amplitude $a \sim \epsilon^{1 / 2} H$. In the radiative core, however, $H \sim R$, so the small-scale mode would have more energy than the large-scale one by a factor of order $\left(a / \delta R_{*}\right)^{2} \sim \epsilon^{-1}$.

Clearly, other nonlinear processes must intervene to halt the growth of the small-scale mode before frequency shifts become important. In the situation contemplated here, we foresee only two such processes: one is the decrease in the parametric growth rate as the energy in the primary mode declines; and the other is the destabilization of granddaughter modes. Both processes have been discussed in $§ 2.2$. 
Table 1

Mode coupling coefficients for quadrupole $\mathrm{f}$ - and g-modes of a solar model with high degree g-modes of half the frequency. First two columns are the number of radial nodes and the frequency of the primary mode $(\alpha)$. Third column is coupling coefficent for parametric decay into identical daughter modes $(\beta)$. Last column gives the fractional surface displacement amplitude of the primary mode when its energy is $10^{42} \mathrm{erg}$.

\begin{tabular}{|c|c|r|c|}
\hline$n_{\alpha}$ (order) & $\begin{array}{c}\nu_{\alpha} \\
(\mu \mathrm{Hz})\end{array}$ & $\begin{array}{c}\kappa_{\alpha \beta \beta} \\
\left(\mathrm{erg}^{-1 / 2}\right)\end{array}$ & $\begin{array}{c}\delta \mathrm{R} / \mathrm{R}_{*} \text { when } \\
\mathrm{E}_{\alpha}=10^{42} \mathrm{erg}\end{array}$ \\
\hline & 372.2 & $4.19 \times 10^{-25}$ & $1.7 \times 10^{-3}$ \\
0 & 300.8 & $-4.43 \times 10^{-25}$ & $1.8 \times 10^{-3}$ \\
1 & 262.7 & $1.13 \times 10^{-24}$ & $1.6 \times 10^{-3}$ \\
2 & 225.7 & $-1.31 \times 10^{-24}$ & $1.1 \times 10^{-3}$ \\
3 & 195.6 & $2.13 \times 10^{-24}$ & $8.7 \times 10^{-4}$ \\
4 & 170.9 & $-2.86 \times 10^{-24}$ & $7.2 \times 10^{-4}$ \\
5 & 151.1 & $4.50 \times 10^{-24}$ & $6.3 \times 10^{-4}$ \\
6 & 135.0 & $-7.00 \times 10^{-24}$ & $6.0 \times 10^{-4}$ \\
7 & 121.9 & $7.86 \times 10^{-24}$ & $5.9 \times 10^{-4}$ \\
8 & 111.0 & $-8.61 \times 10^{-24}$ & $5.7 \times 10^{-4}$ \\
9 & 101.7 & $1.48 \times 10^{-23}$ & $5.8 \times 10^{-4}$ \\
10 & 93.9 & $-1.49 \times 10^{-23}$ & $5.9 \times 10^{-4}$ \\
11 & 87.1 & $2.17 \times 10^{-23}$ & $6.0 \times 10^{-4}$ \\
12 & & &
\end{tabular}




\section{Table 2}

The size of convection zone in main sequence stars of metalicity $(z)$ equal to 0.0001 and age 13.0 Gyr. The first column is the mass of the star in the unit of solar mass, the second column is the effective temperature, the third column is the thickness of the convection zone divided by the radius of the star, and the last column is the fractional mass in the convection zone. These numbers, obtained using the Yale stellar evolution code, were kindly provided by Constantine Deliyannis.

\begin{tabular}{|c|c|c|c|}
\hline $\mathrm{M}_{*}$ & $\mathrm{~T}_{\text {eff }}$ & $\mathrm{R}_{c} / \mathrm{R}_{*}$ & $\mathrm{M}_{c} / \mathrm{M}_{*}$ \\
\hline 0.50 & 4567 & 0.345 & 0.1940 \\
0.65 & 5509 & 0.230 & 0.0274 \\
0.75 & 6227 & 0.135 & 0.0012 \\
\hline
\end{tabular}




\section{REFERENCES}

Christensen-Dalsgaard, J., and Berthomieu G. 1991, in Solar Interior and Atmosphere, eds. Cox, A.N., Livingston, W.C., and Matthews, M.S. (University of Arizona Press)

Deliyannis, C. 1995, personal communication

Dzimbowski, W. 1982, Acta. Astro. 32, 147

Edmonds, A. R. 1960 Angular Momentum in Quantum Mechanics (Princeton:Princeton University Press)

Goldreich, P., Murray, N., \& Kumar, P. 1994, ApJ, 424, 466

Kochanek, C.S. 1992, ApJ, 385, 604

Kumar, P., Ao, C.A., and Quataert, E.J. 1995, ApJ, August 1

Lai, D., Rasio, F.A., and Shapiro, S.L. 1994, ApJ, 423, 344

Landau, L.D., and Lifshitz, E.M. 1976, Mechanics, third ed. (Oxford: Pergamon)

Mardling, R. 1995, to appear in AA

Misner, C.W., Thorne, K.S., and Wheeler, J.A., 1973, Gravitation, Freeman, San Francisco, California

Press, W.H., and Teukolsky, S.A. 1977, ApJ, 213, 183

Pinsonneault, M., Deliyannis, C., and Demarque, P. 1992, ApJS, 78, 179

Stix, M. 1991, The Sun: an introduction (New York: Springer-Verlag) 


\section{Figure Captions}

FIG. 1.- Parametric nonlinear interaction involving $10^{3}$ modes. The amplitude of the primary mode is shown as a function of time.

FIG 2.- The upper panel shows the radial displacement eigenfunction of the f-mode of energy $=10^{45} \mathrm{erg}$ (solid line), and the superposition of radial p-mode displacement-functions generated as a result of nonlinear couplings to the f-mode (dash-dot curve). The lower panel shows the radial derivative of wave functions shown in the upper panel.

FIG. 3. - Energy input per orbit (in erg) into quadrupole f- and a few low order g-modes of $m=\ell=2$, as a function of periastron distance. The mass of the star is $0.7 M_{\odot}$, and the orbital eccentricity is 1.0. The star has been modelled as a polytrope of index 3 with the ratio of specific heat taken to be $5 / 3$. From top to bottom at left $\left(d_{\text {peri }} / R_{*}=2.5\right)$ the modes are $g_{2}, f, g_{4}, g_{6}, g_{8}$, and $g_{10}$.

FIG 4. - The log of nonlinear damping time (in days) for a few low order quadrupole modes of a $0.7 M_{\odot}$ radiative star as a function of periastron distance. The linear radiative damping time for all of these modes are about $10^{4}$ years. Curves correspond to same modes as in Fig. 3: that is, from top to bottom at $d_{\text {peri }} / R_{*}=5, f, g_{2}, g_{4}, g_{6}, g_{8}$, and $g_{10}$. 


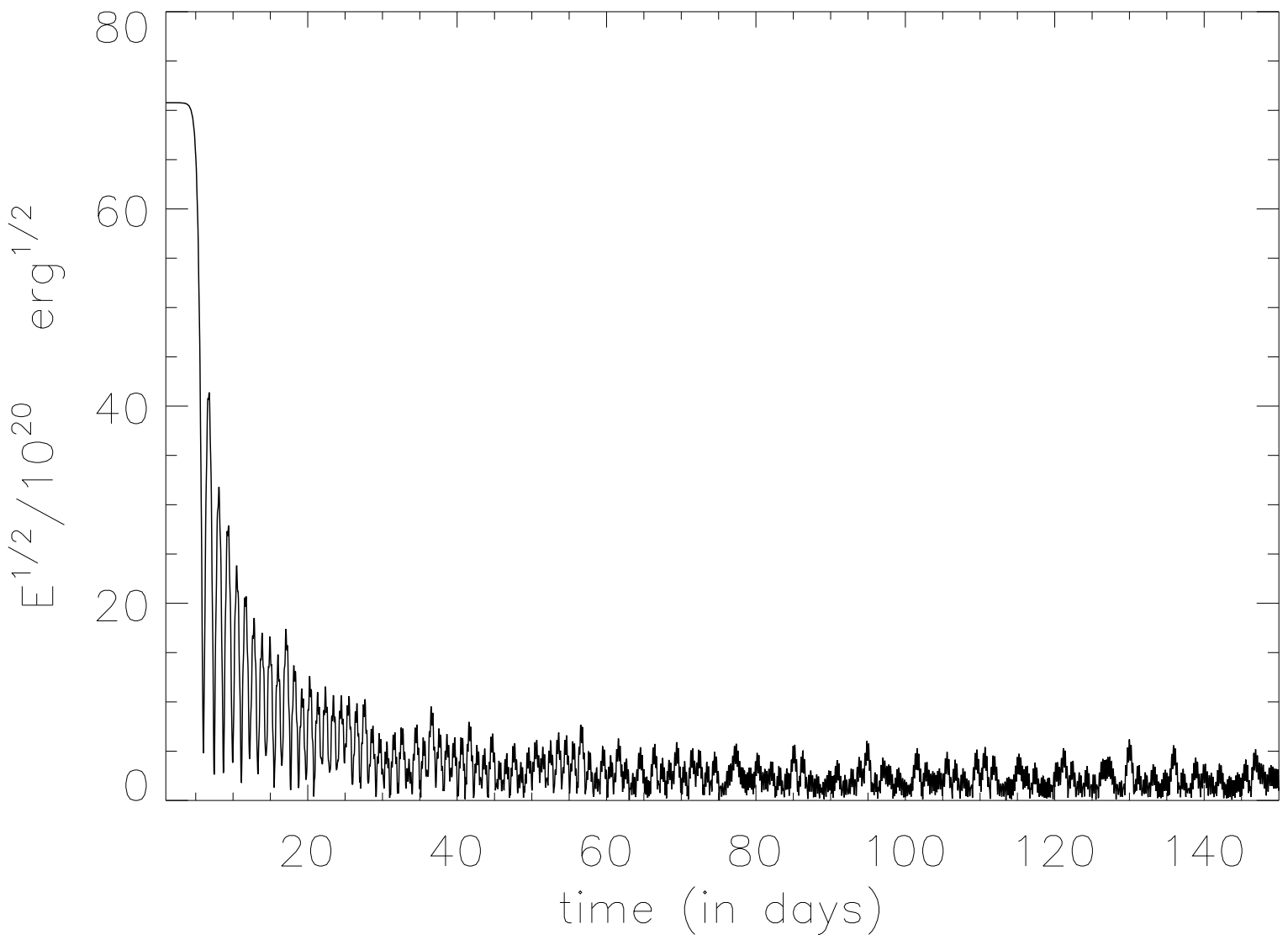



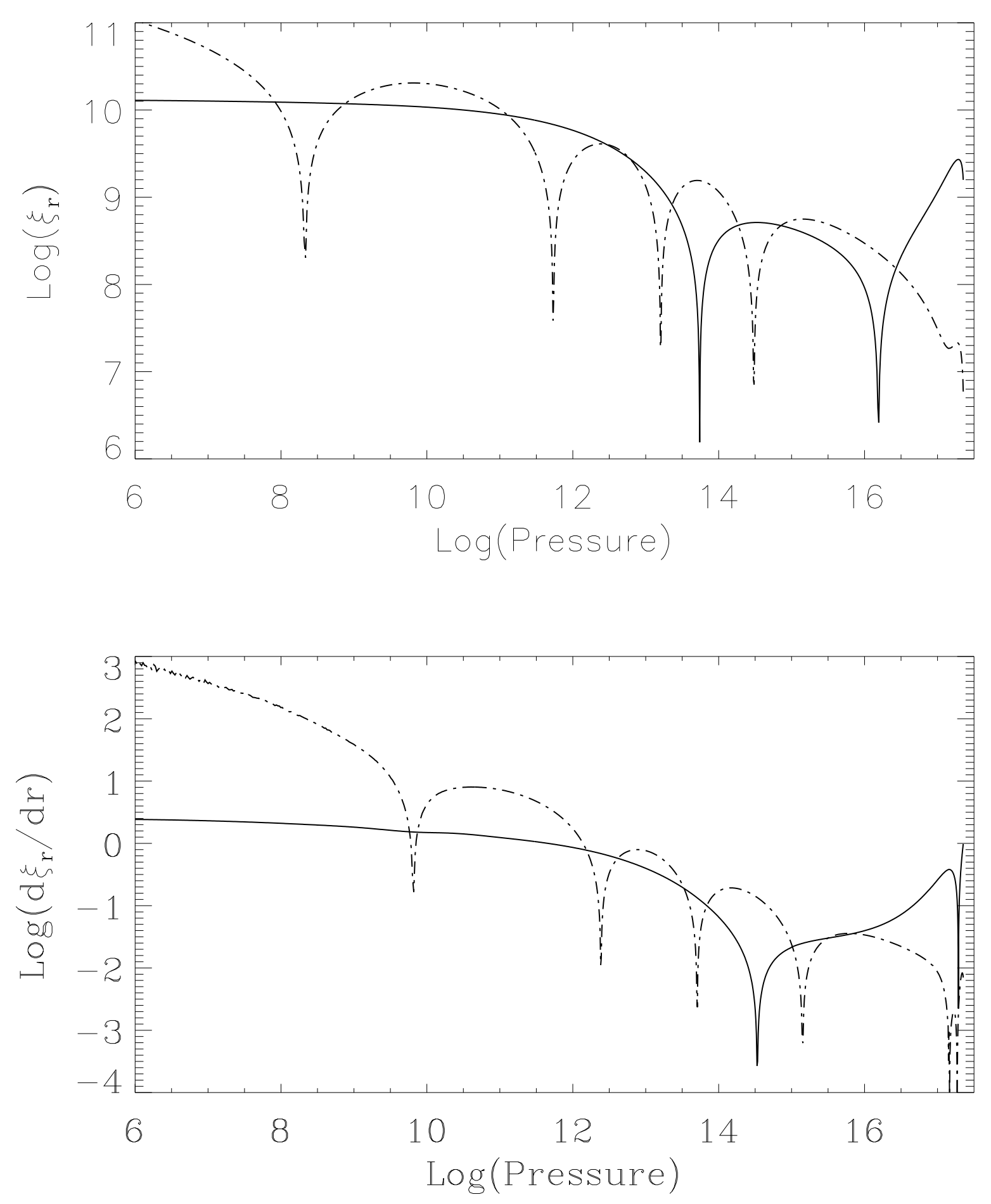


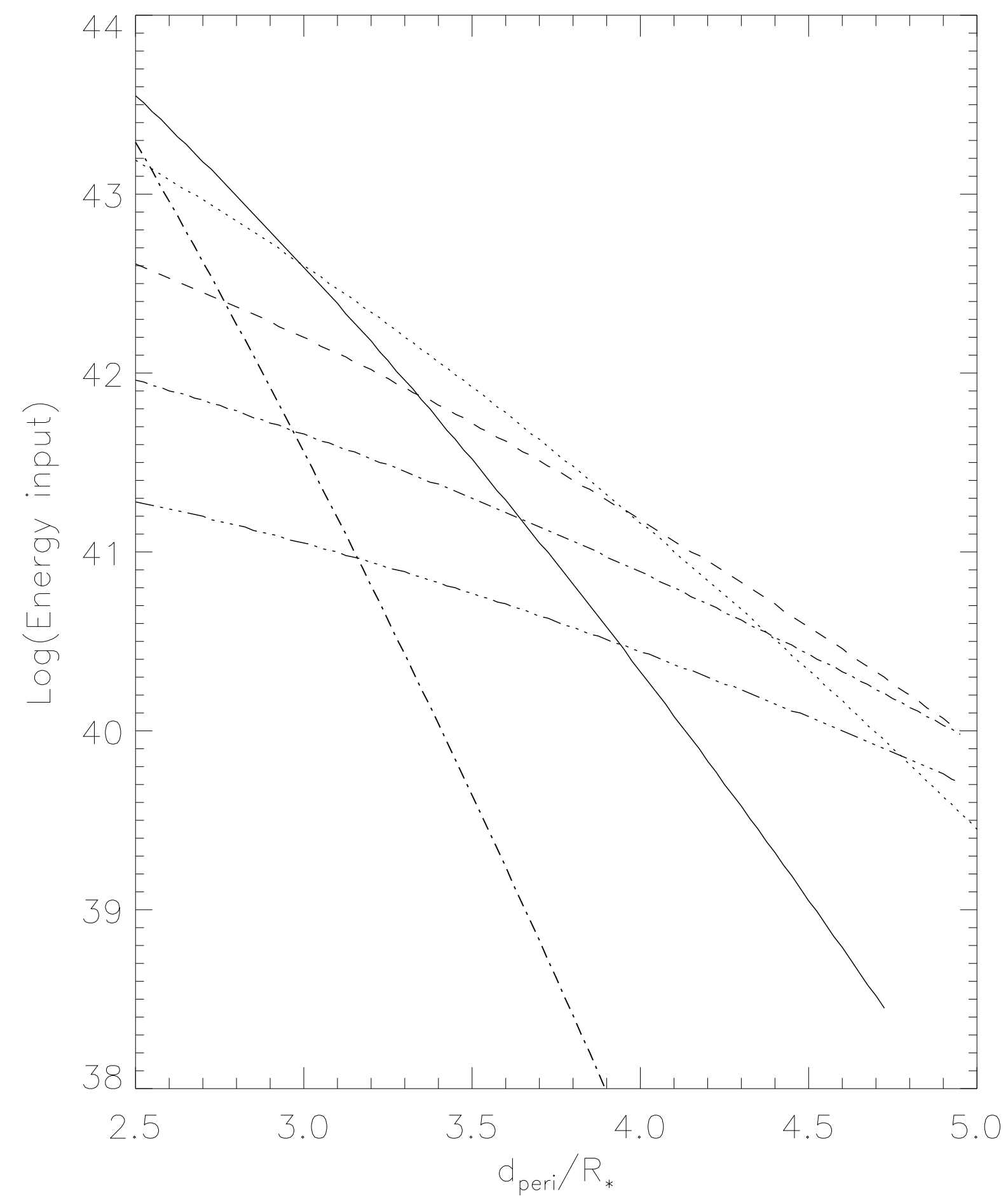




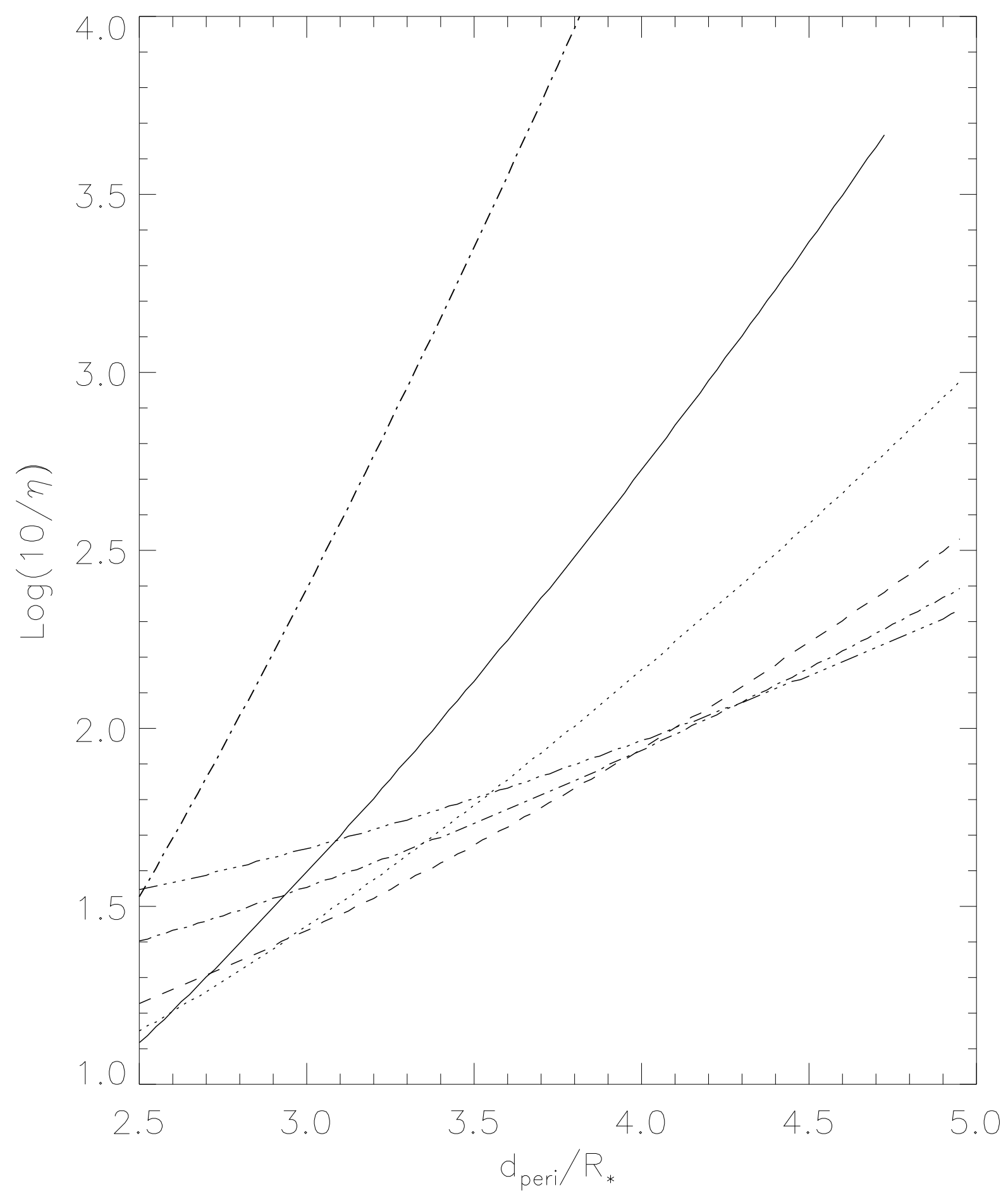

